\title{
Investigation of Continuous-Time Quantum Walk Via Spectral Distribution Associated with Adjacency Matrix
}

\author{
M. A. Jafarizadeh ${ }^{a, b, c *}$, S. Salimi ${ }^{a, b} \dagger$ \\ ${ }^{a}$ Department of Theoretical Physics and Astrophysics, Tabriz University, Tabriz 51664, Iran. \\ ${ }^{b}$ Institute for Studies in Theoretical Physics and Mathematics, Tehran 19395-1795, Iran. \\ ${ }^{c}$ Research Institute for Fundamental Sciences, Tabriz 51664, Iran.
}

July 3, 2018

*E-mail:jafarizadeh@tabrizu.ac.ir

†E-mail:shsalimi@tabrizu.ac.ir 


\begin{abstract}
Using the spectral distribution associated with the adjacency matrix of graphs, we introduce a new method of calculation of amplitudes of continuous-time quantum walk on some rather important graphs, such as line, cycle graph $C_{n}$, complete graph $K_{n}$, graph $G_{n}$, finite path and some other finite and infinite graphs, where all are connected with orthogonal polynomials such as Hermite, Laguerre, Tchebichef and some other orthogonal polynomials. It is shown that using the spectral distribution, one can obtain the infinite time asymptotic behavior of amplitudes simply by using the method of stationary phase approximation(WKB approximation), where as an example, the method is applied to star, two-dimensional comb lattices, infinite Hermite and Laguerre graphs. Also by using the Gauss quadrature formula one can approximate infinite graphs with finite ones and vice versa, in order to derive large time asymptotic behavior by WKB method. Likewise, using this method, some new graphs are introduced, where their amplitude are proportional to product of amplitudes of some elementary graphs, even though the graphs themselves are not the same as Cartesian product of their elementary graphs. Finally, via calculating mean end to end distance of some infinite graphs at large enough times, it is shown that continuous time quantum walk at different infinite graphs belong to different universality classes which are also different than those of the corresponding classical ones.
\end{abstract}

Keywords: Quantum walk, Continuous-time quantum walk, Spectral distribution, Graph.

PACs Index: 03.65.Ud 


\section{Introduction}

The theory of Markov chains and random walks on graphs is fundamental to mathematics, physics, and computer science $[1,2,3]$ as it provides a beautiful mathematical framework to study the stochastic process and its applications. Among the known examples of the applications we can mention Monte Carlo methods in statistics, the theory of diffusion in statistical physics, and algorithmic techniques for sampling and random generation of combinatorial structures in computer science (based on rapid mixing of certain Markov chains). Two pervasive algorithmic ideas in quantum computation are Quantum Fourier Transform (QFT) and amplitude amplification (see [4]). Most subsequent progress in quantum computing owed much to these two beautiful ideas. But there are many problems whose characteristics match neither the QFT nor the amplitude amplification mold (e.g., the graph isomorphism problem), where this begs for new additional tools to be discovered.

A natural way to discover new quantum algorithmic ideas is to adapt a classical one to the quantum model. An appealing well-studied classical idea in statistics and computer science is the method of random walks [5]. Recently, the quantum analogue of classical random walks has been studied in a flurry of works $[6,7,8,9,10,11]$. The works of Moore and Russell [10] and Kempe [11] showed faster bounds on instantaneous mixing and hitting times for discrete and continuous quantum walks on a hypercube (compared to the classical walk).

A study of quantum walks on simple lattice is well known in physics(see [12]). Recent studies of quantum walks on more general graphs were described in $[6,7,9,13,14]$. Some of these works studies the problem in the important context of algorithmic problems on graphs and suggests that quantum walks is a promising algorithmic technique for designing future quantum algorithms.

Several important classes of graphs studied in classical random walks include the binary $n$-cube, the circulant graphs, and the group-theoretic Cayley graphs. The binary $n$-cube and 
circulant graphs are important in the study of interconnection networks and complexity of Boolean function, and Cayley graphs capture strong gorup-theortic ingredients of important problems, such as graph isomorphism. Since most of these graphs are regular, the classical random walks on them are known to converge or to mix towards the uniform stationary distribution. The mixing properties of continuous-time quantum walks on the same graphs were found to exhibit non-classical behavior $[10,15,16,17]$.

Here in this work we have used spectral distribution associated with the adjacency matrix, of some particular graphs which possess quantum distribution ( for more details see Ref.[19]) in studying continuous-time quantum walk. Spectral distribution helps us to give a general expression for the amplitude of observing the continuous-time quantum walker on a given site in terms of the integral over product of the spectral distribution and some polynomials (in most cases over the well-known orthogonal polynomial; for the details see the appendix II). We use isometry between the orthogonal polynomials (Hermite, Laguerre and Tchebichef) and interacting Fock space associated with the infinite graphs, representing raising (lowering) part adjacency matrix, with first order differential operator, and obtain the amplitude probability for infinite graphs associated to orthogonal polynomials. In obtaining the amplitude probability for many of the finite (complete $K_{n}$, complete cycle, $G_{n}$, finite path)or infinite graphs(line, associated graphs of orthogonal polynomials Hermite and Laguerre) we also study the behavior of graph for large vertices, and the asymptotic behavior of the walks. By using direct product graphs, we obtain the new graphs and define an approach for calculating the amplitude probability from the subgraphs. Also by using the Gauss quadrature formula one can approximate infinite graphs with finite ones and vice versa, which leads to the derivation of large time asymptotic behaviorcontinuous-time quantum walk simply by using the method of stationary phase. Also, we introduce graphs which have different structure, but they have uniform amplitude for observing particle at every level. At the end, via calculating average visiting strata( sites for infinite line) which is equivalent to mean end to end distance of some 
infinite graphs at large enough times, it is shown that continuous time quantum walk on infinite Hermite and line graphs belong to the same universality class which is different from that of infinite Laguerre one.

The organization of this paper is as follows. In section 2, we give a brief outline of graphs and their adjacency matrices. In Section 3, we review the quantum decomposition for adjacency matrix of graphs, and the method for obtaining vacuum spectral distribution $\mu$, and give some examples of isometry from orthogonal polynomials into interacting Fock space. Section 4 is devoted to the method of computing amplitude probability for continuous-time quantum walk, through spectral distribution $\mu$ of the adjacency matrix $A$. Section 5 is concerned with direct product of quantum decompositions for finite graphs. In section 6 , we calculate the amplitude probability for continuous-time quantum walk on bunches of finite and infinite graphs. In section 7 , in first subsection we evaluate the average visiting strata of some infinite graphs for large enough time, and in remaining subsections, large time asymptotic behavior of quantum walks on different finite and infinite graphs of section 6 are obtained by using the method of stationary phase(WKB) and Gauss quadrature rule. Paper is ended with a brief conclusion and two appendices $I$ and $I I$, where the first appendix consists of the proof of lemma regarding the equality of the amplitudes associated with vertices belonging to the same stratum and the second appendix contains the list of some of the orthogonal polynomials connected with some particular infinite and finite graphs, respectively.

\section{Graphs and its adjacency matrix}

In this section we give a brief outline of some of the main features of graphs and walk on them, such as adjacency matrix, stratification and orthonormal basis of strata.

A graph is a pair $G=(V, E)$, where $V$ is a non-empty set and $E$ is a subset of $\{(i, j) ; i, j \in$ $V, i \neq j\}$. Elements of $V$ and of $E$ are called vertices and edges, respectively. Two vertices 
$i ; j \in V$ are called adjacent if $(i, j) \in E$, and in that case we write $i \sim j$. For a graph $G=(V, E)$ we define the adjacency matrix $A=\left(A_{i j}\right)_{i, j \in V}$ by

$$
A_{i j}= \begin{cases}1 & \text { if } i \sim j \\ 0 & \text { otherwise. }\end{cases}
$$

Obviously, (i) $A$ is symmetric; (ii) an element of $A$ takes a value in $\{0,1\}$; (iii) a diagonal element of $A$ vanishes. Conversely, for a non-empty set $V$, a graph structure is uniquely determined by such a matrix indexed by $V$. The degree or valency of a vertex $i \in V$ is defined by

$$
\kappa(i)=|\{j \in V ; i \sim j\}|,
$$

where $\mid$. $\mid$ denotes the cardinality. A finite sequence $i_{0} ; i_{1} ; \ldots ; i_{n} \in V$ is called a walk of length $n$ (or of $n$ steps) if $i_{k-1} \sim i_{k}$ for all $k=1,2, \ldots, n$. In a walk some vertices may occur repeatedly. Unless otherwise stated, we always assume that a graph under discussion satisfies:

(a) (connectedness) any pair of distinct vertices are connected by a walk;

(b) (local boundedness) $\kappa(i)<\infty$ for all $i \in V$; In fact, the examples in this paper satisfy the following condition which is stronger than (b):

$\left(b^{\prime}\right)$ (uniform boundedness) $\sup _{i \in V} \kappa(i)<\infty$.

Let $l^{2}(V)$ denote the Hilbert space of C-valued square-summable functions on $\mathrm{V}$, and $\{|i\rangle ; i \in$ $V\}$ becomes a complete orthonormal basis of $l^{2}(V)$. The adjacency matrix is considered as an operator acting in $l^{2}(V)$ in such a way that

$$
A|i\rangle=\sum_{i \sim j}|j\rangle, \quad i \in V
$$

Then, $A$ becomes a self-adjoint operator equipped with a natural domain. As is easily checked, $\left(b^{\prime}\right)$ is a necessary and sufficient condition for $A$ to be a bounded operator on $l^{2}(V)$. 


\subsection{Stratification}

For $i \neq j$ let $\partial(i, j)$ be the length of the shortest walk connecting $i$ and $j$. By definition $\partial(i, j)=0$ for all $i \in V$. The graph becomes a metric space with the distance function $\partial$. Note that $\partial(i, j)=1$ if and only if $i \sim j$. We fix a point $o \in V$ as an origin of the graph. Then, the graph is stratified into a disjoint union of strata:

$$
V=\bigcup_{k=0}^{\infty} V_{k}, \quad V_{k}=\{i \in V ; \partial(o, i)=k\} .
$$

With each stratum $V_{k}$ we associate a unit vector in $l^{2}(V)$ defined by

$$
\left|\phi_{k}\right\rangle=\frac{1}{\sqrt{\left|V_{k}\right|}} \sum_{i \in V_{k}}|k, i\rangle,
$$

where, $|k, i\rangle$ denotes the eigenket of $i$ th vertex at the stratum $k$. The closed subspace of $l^{2}(V)$ spanned by $\left\{\left|\phi_{k}\right\rangle\right\}$ is denoted by $\Gamma(G)$. Since $\left\{\left|\phi_{k}\right\rangle\right\}$ becomes a complete orthonormal basis of $\Gamma(G)$, we often write

$$
\Gamma(G)=\sum_{k} \oplus \mathbf{C}\left|\phi_{k}\right\rangle
$$

\section{Quantum decomposition}

In this section, first we review the quantum decomposition for adjacency matrix of some particular graphs called QD graphs, and the method for obtaining the vacuum spectral distribution $\mu$ (for more detail see Ref. [18]), then we give some examples of isometry from orthogonal polynomials into interacting Fock space.

Let $A$ be the adjacency matrix of a graph $G=(V, E)$. According to the stratification (2-1), we define three matrices $A^{+}, A^{-}$and $A^{0}$ as follows: for $i \in V_{k}$ we set

$$
\left(A^{+}\right)_{j i}= \begin{cases}A_{j i} & \text { if } j \in V_{k+1} \\ 0 & \text { otherwise. }\end{cases}
$$




$$
\begin{aligned}
& \left(A^{-}\right)_{j i}= \begin{cases}A_{j i} & \text { if } j \in V_{k-1} \\
0 & \text { otherwise. }\end{cases} \\
& \left(A^{0}\right)_{j i}= \begin{cases}A_{j i} & \text { if } j \in V_{k} \\
0 & \text { otherwise. }\end{cases}
\end{aligned}
$$

Or equivalently, for $|k, i\rangle$,

$$
A^{+}|k, i\rangle=\sum_{j \in V_{k+1}}|k+1, j\rangle, \quad A^{-}|k, i\rangle=\sum_{j \in V_{k-1}}|k-1, j\rangle, \quad A^{0}|k, i\rangle=\sum_{j \in V_{k}}|k, j\rangle,
$$

for $j \sim i$. Since $i \in V_{k}$ and $i \sim j$ then $j \in V_{k-1} \cup V_{k} \cup V_{k+1}$, where we tacitly understand that $V_{-1}=\emptyset$, Now with the help of [19] we define

$$
A=A^{+}+A^{-}+A^{0}
$$

This is called quantum decomposition of $A$ associated with the stratification (2-1). Note also that

$$
\left(A^{+}\right)^{\star}=A^{-}, \quad\left(A^{0}\right)^{\star}=A^{0},
$$

which can be verified easily. The vector state corresponding to $|o\rangle=\left|\phi_{0}\right\rangle$, with $o \in V$ as the fixed origin, is analogous to the vacuum state in Fock space. According to Ref.[19], the $<A^{m}>$ coincides with the number of $m$-step walks starting and terminating at $o$, also, by lemma 2.2, [19] if $\Gamma(G)$ is invariant under the quantum components $A^{\varepsilon}, \varepsilon \in\{+,-, 0\}$, then there exist two Szegö- Jacobi sequences $\left\{\omega_{k}\right\}_{k=1}^{\infty}$ and $\left\{\alpha_{k}\right\}_{k=1}^{\infty}$ derived from $A$, such that

$$
\begin{gathered}
A^{+}\left|\phi_{k}\right\rangle=\sqrt{\omega_{k+1}}\left|\phi_{k+1}\right\rangle, \quad k \geq 0 \\
A^{-}\left|\phi_{0}\right\rangle=0, \quad A^{-}\left|\phi_{k}\right\rangle=\sqrt{\omega_{k}}\left|\phi_{k-1}\right\rangle, \quad k \geq 1 \\
A^{0}\left|\phi_{k}\right\rangle=\alpha_{k+1}\left|\phi_{k}\right\rangle, \quad k \geq 0,
\end{gathered}
$$

where $\sqrt{\omega_{k}}=\frac{\left|V_{k+1}\right|^{1 / 2}}{\left|V_{k}\right|^{1 / 2}} \kappa_{-(j)}, \kappa_{-(j)}=\left|\left\{i \in V_{k} ; i \sim j\right\}\right|$ for $j \in V_{k+1}$ and $\alpha_{k+1}=\kappa_{0(j)}$, such that $\kappa_{0(j)}=\left|\left\{i \in V_{k} ; i \sim j\right\}\right|$ for $j \in V_{k}$. 
Obviously from the relation between $\omega_{k}, \alpha_{k}, \kappa_{-}(y)$ and $\left|V_{k}\right|$ it follows that $\left|V_{k}\right|-1 \geq \alpha_{k+1}$ and $\frac{\left|V_{k}\right| \alpha_{k+1}}{2}$ must be integer.

From now on we call the graphs with the above property as kids of graphs which possess quantum distribution or QD type.

\section{Spectral distribution $\mu$ of the adjacency matrix $A$}

The spectral properties of the adjacency matrix of a graph play an important role in many branches of mathematics and physics. The spectral distribution can be generalized in various ways. In this work, following Ref.[19], we consider the spectral distribution $\mu$ of the adjacency matrix $A$ :

$$
<A^{m}>=\int_{R} x^{m} \mu(d x), \quad m=0,1,2, \ldots
$$

where $\left\langle.>\right.$ is the mean value with respect to a state $\left.\mid \phi_{0}\right\rangle$ (the ket corresponds to the ground stratum). By the local boundedness condition (b) of section 3 the moment sequence $\left\{<A^{m}>\right.$ \}$_{m=0}^{\infty}$ is well-defined[19]. Then the existence of a spectral distribution satisfying (4-10) is a consequence of Hamburgers theorem, see e.g., Shohat and Tamarkin [[21], Theorem 1.2].

We may apply the canonical isomorphism from the interacting Fock space onto the closed linear span of the orthogonal polynomials determined by the Szegö-Jacobi sequences $\left(\left\{\omega_{k}\right\},\left\{\alpha_{k}\right\}\right)$. More precisely, the spectral distribution $\mu$ under question is characterized by the property of orthogonalizing the polynomials $\left\{Q_{n}\right\}$ defined recurrently by

$$
\begin{gathered}
Q_{0}(x)=1, \quad Q_{1}(x)=x-\alpha_{1}, \\
x Q_{n}(x)=Q_{n+1}(x)+\alpha_{n+1} Q_{n}(x)+\omega_{n} Q_{n-1}(x),
\end{gathered}
$$

for $n \geq 1$. If such a spectral distribution is unique (e.g., if the uniform boundedness condition $\left(b^{\prime}\right)$ is fulfilled), the spectral distribution $\mu$ is determined by the identity:

$$
G_{\mu}(x)=\int_{R} \frac{\mu(d y)}{x-y}=\frac{1}{x-\alpha_{1}-\frac{\omega_{1}}{x-\alpha_{2}-\frac{\omega_{2}}{x-\alpha_{3}-\frac{\omega_{3}}{x-\alpha_{4}-\cdots}}}}=\frac{Q_{n-1}^{(1)}(x)}{Q_{n}(x)}=\sum_{l=1}^{n} \frac{A_{l}}{x-x_{l}},
$$


where $G_{\mu}(x)$ is called the Stieltjes transform and $A_{l}$ is the coefficient in the Gauss quadrature formula corresponding to the roots $x_{l}$ of polynomial $Q_{n}(x)$ and where polynomials $\left\{Q_{n}^{(1)}\right\}$ are defined recurrently as

$Q_{0}^{(1)}(x)=1$,

$Q_{1}^{(1)}(x)=x-\alpha_{2}$,

$x Q_{n}^{(1)}(x)=Q_{n+1}^{(1)}(x)+\alpha_{n+2} Q_{n}^{(1)}(x)+\omega_{n+1} Q_{n-1}^{(1)}(x)$,

for $n \geq 1$.

Now if $G_{\mu}(x)$ is known, then the spectral distribution $\mu$ can be recovered from $G_{\mu}(x)$ by means of the Stieltjes inversion formula:

$$
\mu(y)-\mu(x)=-\frac{1}{\pi} \lim _{v \longrightarrow 0^{+}} \int_{x}^{y} \operatorname{Im}\left\{G_{\mu}(u+i v)\right\} d u
$$

Substituting the right hand side of (4-12) in (4-13), the spectral distribution can be determined in terms of $x_{l}, l=1,2, \ldots$, the roots of the polynomial $Q_{n}(x)$, and Guass quadrature constant $A_{l}, l=1,2, \ldots$ as

$$
\mu=\sum_{l} A_{l} \delta\left(x-x_{l}\right)
$$

( for more details see Ref. [18, 20, 21, 22].)

In the following, we show the above mentioned isometry $U$ from the orthogonal polynomial into interacting Fock space in infinite graphs associated with Hermite, Laguerre and Tchebichef polynomials, simply by replacing the raising (lowering) part of adjacency matrix, i.e., $A^{+}\left(A^{-}\right)$ by the corresponding first order differential operators $B_{-}(n)\left(A_{-}(n)\right)$ introduced by one of the authors in Ref. [23] with the following recursion relations

$$
B_{-}(n) Q_{n-1}(x)=Q_{n}(x) ; \quad A_{-}(n) Q_{n}(x)=E(n) Q_{n-1}(x)
$$

where $E(n)=n$, for Hermite, $E(n)=n(n-\gamma), \gamma>-1$, for Laguerre and $E(n)=n^{2}$ for Tchebichef. Now by assuming the canonical isometry $U Q_{n}(x)=\sqrt{\beta_{n}}\left|\phi_{n}\right\rangle$ between the corresponding Hilbert spaces, $A^{+}=U B_{-}(n) U^{\star}, A^{-}=U A_{-}(n) U^{\star}$ and the corresponding operators 
$\omega_{n}=E(n)$, we have

for Laguerre polynomial $Q_{n}(x)=L_{n}(x)$

$$
A^{+}=U\left(-x \frac{d}{d x}+x-n\right) U^{\star} ; \quad A^{-}=U\left(x \frac{d}{d x}-n\right) U^{\star}
$$

for Hermite polynomial $Q_{n}(x)=H_{n}(x)$

$$
A^{+}=U\left(-\frac{d}{d x}+x\right) U^{\star} ; \quad A^{-}=U\left(\frac{d}{d x}\right) U^{\star}
$$

for Tchebichef polynomial $Q_{n}(x)=T_{n}(x)$

$$
A^{+}=U\left(-\left(1-x^{2}\right) \frac{d}{d x}+n x\right) U^{\star} ; \quad A^{-}=U\left(\left(1-x^{2}\right) \frac{d}{d x}-n x\right) U^{\star}
$$

Finally, using the quantum decomposition relations $(3-7,8,9)$ and the recursion relation (4-11) of polynomial $Q_{n}(x)$, the other matrix elements $\left\langle\phi_{k}\left|A^{m}\right| \phi_{0}\right\rangle$ can be written as

$$
\left\langle\phi_{k}\left|A^{m}\right| \phi_{0}\right\rangle=\frac{1}{\sqrt{\omega_{1} \omega_{2} \cdots \omega_{k}}} \int_{R} x^{m} Q_{k}(x) \mu(d x), \quad m=0,1,2, \ldots
$$

Our main goal in this paper is the evaluation of amplitude probability for continuous-time quantum walk by using Eq.(4-16) such that we have

$$
q_{k}(t)=\left\langle\phi_{k}\left|e^{-i A t}\right| \phi_{0}\right\rangle=\frac{1}{\sqrt{\omega_{1} \omega_{2} \cdots \omega_{k}}} \int_{R} e^{-i x t} Q_{k}(x) \mu(d x),
$$

where $q_{k}(t)$ is the amplitude of observing the particle at level $k$ at time $t$. The conservation of probability $\sum_{k=0}\left|q_{k}(t)\right|^{2}=1$ follows immediately from Eq.(4-17) by using the completeness relation of orthogonal polynomials $Q_{n}(x)$. Also by using Eq.(4-17) and (3-7,3-8,3-9), we obtain the following recurrence relation for the amplitude $p_{k}(t)$ :

$$
i \frac{d q_{k}(t)}{d t}=\sqrt{\omega_{k+1}} q_{k+1}(t)+\alpha_{k+1} q_{k}(t)+\sqrt{\omega_{k}} q_{k-1}(t)
$$

Obviously evaluation of $q_{k}(t)$ leads to the determination of the amplitudes at sites belonging to the stratum $V_{k}$, as it is proved in the appendix I, the walker has the same amplitude at 
sites belonging to the same stratum, i.e., we have $q_{i k}(t)=\frac{q_{k}(t)}{\left|V_{k}\right|}, i=0,1, \ldots,\left|V_{k}\right|$, where $q_{i k}(t)$ denotes the amplitude walker at $i$ th site of $k$ th stratum.

Obviously for finite graphs, the formula (4-17) yields

$$
q_{k}(t)=\frac{1}{\sqrt{\omega_{1} \omega_{2} \cdots \omega_{k}}} \sum_{l} A_{l} e^{-i x_{l} t} Q_{k}\left(x_{l}\right),
$$

where by straightforward calculation one can evaluate the average probability for the finite graphs as

$$
P(k)=\lim _{T \rightarrow \infty} \frac{1}{T} \int_{0}^{T}\left|q_{k}(t)\right|^{2} d t=\frac{1}{\omega_{1} \omega_{2} \cdots \omega_{k}} \sum_{l} A_{l}^{2} Q_{k}^{2}\left(x_{l}\right) .
$$

Finally using Gauss quadrature formula [20]

$$
\int f(x) \mu(d x)=\sum_{l=1}^{n} A_{k} f\left(x_{l}\right)
$$

where the constants $A_{l}$ and $x_{l}$ are the same as those appearing in formula(4-12), we can approximate an infinite QD graph corresponding to the sequence of orthogonal polynomials $\left\{Q_{0}, Q_{1}, Q_{2}, \ldots\right\}$, with the finite $n$-stratum QD graph of spectral distribution obtained from Stieltjes transform (4-12) with constant $A_{k}$ and $x_{k}$, and vice versa. Therfore, using Gauss quadrature formula, we can interchange formulas (4-17) and (4-19) of finite and infinite graphs. This approximation can be very useful in obtaining the large time asymptotic behavior of quantum walk on graphs with finitely many vertices.

Since, studying the large time behavior of quantum walks on finite or infinite graphs by this method, naturally leads us to consider the behavior of integrals of the form

$$
I(t)=\int_{a}^{b} e^{-i t \phi(x)} g(x) d x \quad a \leq x \leq b
$$

as $t$ tends to infinity. There is a well-developed theory of the asymptotic expansion of integrals known as method of stationary phase approximation or WKB [24, 25], which allows us to determine, very precisely, the leading terms in the expansion of the integral in terms of simple functions of $t$ (such as inverse powers of $t$ ). In section 7 we will study asymptotic behavior of some finite and infinite graphs at distant time by using Gauss quadrature formula and WKB approximation. 


\section{$5 \quad$ The direct product of QD finite graphs}

In this section, we study continuous-time quantum walk on direct product of QD finite graphs. Let $G_{i}, i=1,2, . ., n$ be graphs of finite vertices with the corresponding adjacency matrices $A_{i}, i=1,2, \ldots, n$. Then their direct product

$$
G_{1} \otimes \cdots \otimes G_{n}
$$

is a graph with the following adjacency matrix $A$ :

$$
A=\sum_{j=1}^{n} I \otimes \cdots \otimes A_{j} \otimes \cdots \otimes I
$$

where the $j-t h$ term in the sum has $A_{j}$ appearing in the $j-t h$ place in the tensor product.

In this case, for the amplitude of the ground state $\left|\phi_{0}\right\rangle=\left|\phi_{0}^{(1)}\right\rangle\left|\phi_{0}^{(2)}\right\rangle \cdots\left|\phi_{0}^{(n)}\right\rangle$, similar to Eq.(4-10), we have

$$
\left\langle\phi_{0}\left|A^{m}\right| \phi_{0}\right\rangle=\int \cdots \int\left(x_{1}+x_{2}+\cdots+x_{n}\right)^{m} \mu\left(d x_{1}\right) \mu\left(d x_{2}\right) \cdots \mu\left(d x_{n}\right) .
$$

Thus the amplitude of the ground state can be written as

$$
\begin{gathered}
q_{0}(t)=\left\langle\phi_{0}\left|e^{-i t A}\right| \phi_{0}\right\rangle=\int \cdots \int e^{-i t x_{1}} e^{-i t x_{2}} \cdots e^{-i t x_{n}} \mu\left(d x_{1}\right) \mu\left(d x_{2}\right) \cdots \mu\left(d x_{n}\right) \\
=\left\langle\phi_{0}^{(1)}\left|e^{-i t A_{1}}\right| \phi_{0}^{(1)}\right\rangle\left\langle\phi_{0}^{(2)}\left|e^{-i t A_{2}}\right| \phi_{0}^{(2)}\right\rangle \cdots\left\langle\phi_{0}^{(n)}\left|e^{-i t A_{n}}\right| \phi_{0}^{(n)}\right\rangle .
\end{gathered}
$$

Therefore the amplitude of the walker at ground state $q_{0}(t)\left(q_{k}(t)\right.$ the amplitude of the walker at stratum $k$ and time $t$ ) of product graph can be obtained simply by multiplying the corresponding amplitudes of sub-graphs. Also comparing equations (4-17) and (5-26) one can determine the spectral distribution $\mu(d x)$ and Stieltjes transform $G_{\mu}(x)$ (consequently all amplitudes of product graph), provided that the product graph possesses quantum distribution, too. In the following, we will obtain the required conditions for obtaining QD graphs from the product of two given QD graphs. As we will see it is quite possible to obtain QD graphs with the same but non-isomorphic spectral distribution (having different adjacency matrices, such 
that the adjacency matrix of product one may not be tensor product of adjacency matrix of multiplicand graphs) from the products of QD graphs.

Considering two QD graphs, $G_{1}$ with $\omega_{1}^{1}, \omega_{2}^{1}, \ldots, \omega_{n}^{1} ; \alpha_{2}^{1}, \alpha_{3}^{1}, \ldots, \alpha_{n+1}^{1}$ and $G_{2}$ with $\omega_{1}^{2}, \omega_{2}^{2}, \ldots \omega_{m}^{2} ; \alpha_{2}^{2}, \alpha_{3}^{2}, \ldots, \alpha_{m+1}^{2}$, we obtain the following QD graph from their product

$$
\begin{gathered}
\left|\phi_{0}\right\rangle=\left|\phi_{0}^{1}\right\rangle\left|\phi_{0}^{2}\right\rangle, \\
\left|\phi_{1}\right\rangle=\frac{1}{\sqrt{\omega_{1}^{1}+\omega_{1}^{2}}}\left(\sqrt{\omega_{1}^{1}}\left|\phi_{1}^{1}\right\rangle\left|\phi_{0}^{2}\right\rangle+\sqrt{\omega_{1}^{2}}\left|\phi_{0}^{1}\right\rangle\left|\phi_{1}^{2}\right\rangle\right), \\
\left|\phi_{2}\right\rangle=\frac{1}{\sqrt{\omega_{1}^{1} \omega_{2}^{1}+4 \omega_{1}^{1} \omega_{2}^{2}+\omega_{1}^{2} \omega_{2}^{2}}}\left(\sqrt{\omega_{1}^{1} \omega_{2}^{1}}\left|\phi_{2}^{1}\right\rangle\left|\phi_{0}^{2}\right\rangle+2 \sqrt{\omega_{1}^{1} \omega_{1}^{2}}\left|\phi_{1}^{1}\right\rangle\left|\phi_{1}^{2}\right\rangle+\sqrt{\omega_{1}^{2} \omega_{2}^{2}}\left|\phi_{0}^{1}\right\rangle\left|\phi_{2}^{2}\right\rangle\right), \\
\vdots \\
\left|\phi_{m n-1}\right\rangle=\left|\phi_{n}^{1}\right\rangle\left|\phi_{m}^{2}\right\rangle,
\end{gathered}
$$

with

$$
\begin{gathered}
\omega_{1}=\omega_{1}^{1}+\omega_{1}^{2}, \quad \omega_{2}=\frac{\omega_{1}^{1} \omega_{2}^{2}+4 \omega_{1}^{1} \omega_{1}^{2}+\omega_{1}^{2} \omega_{2}^{2}}{\omega_{1}^{1}+\omega_{1}^{2}}, \\
\omega_{3}=\frac{\omega_{1}^{1} \omega_{2}^{1} \omega_{3}^{1}+9\left(\omega_{1}^{1} \omega_{2}^{1} \omega_{1}^{2}+\omega_{1}^{1} \omega_{1}^{2} \omega_{2}^{2}\right)+\omega_{1}^{2} \omega_{2}^{2} \omega_{3}^{2}}{\omega_{1}^{1} \omega_{2}^{2}+4 \omega_{1}^{1} \omega_{1}^{2}+\omega_{1}^{2} \omega_{2}^{2}}, \\
\omega_{4}=\frac{\omega_{1}^{1} \omega_{2}^{1} \omega_{3}^{1} \omega_{4}^{1}+16 \omega_{1}^{1} \omega_{2}^{1} \omega_{3}^{1} \omega_{1}^{2}+36 \omega_{1}^{1} \omega_{2}^{1} \omega_{1}^{2} \omega_{2}^{2}+16 \omega_{1}^{1} \omega_{1}^{2} \omega_{2}^{2} \omega_{3}^{2}+\omega_{1}^{2} \omega_{2}^{2} \omega_{3}^{2} \omega_{4}^{2}}{\omega_{1}^{1} \omega_{2}^{1} \omega_{3}^{1}+9\left(\omega_{1}^{1} \omega_{2}^{1} \omega_{1}^{2}+\omega_{1}^{1} \omega_{1}^{2} \omega_{2}^{2}\right)+\omega_{1}^{2} \omega_{2}^{2} \omega_{3}^{2}}, \ldots \\
\alpha_{2}=\alpha_{2}^{1}=\alpha_{2}^{2}, \quad \alpha_{3}=\alpha_{2}^{1}+\alpha_{2}^{2}=\alpha_{3}^{1}=\alpha_{3}^{2}, \\
\alpha_{4}=\alpha_{2}^{1}+\alpha_{3}^{2}=\alpha_{3}^{1}+\alpha_{2}^{2}=\alpha_{4}^{1}=\alpha_{4}^{2}, \ldots, \alpha_{k+1}=k \alpha_{2}^{1} \ldots . .
\end{gathered}
$$

Now, by acting operators $A^{+}, A^{-}$and $A_{0}$ on the quantum state of the product graph (5-23), we get the following required conditions between the QD parameters of multiplicant graphs in order to obtain a QD graph from their Cartesian product

$$
\begin{gathered}
\omega_{2}^{1}+2 \omega_{1}^{2}=2 \omega_{1}^{1}+\omega_{2}^{2}, \quad 2\left(\omega_{3}^{1}+3 \omega_{1}^{2}\right)=3\left(\omega_{2}^{1}+\omega_{2}^{2}\right), \\
\omega_{3}^{1}+3 \omega_{1}^{2}=3 \omega_{1}^{1}+\omega_{3}^{2}, \quad \omega_{4}^{1}+4 \omega_{1}^{2}=4 \omega_{1}^{1}+\omega_{4}^{2}, \\
3 \omega_{4}^{1}+12 \omega_{1}^{2}=4 \omega_{3}^{1}+6 \omega_{2}^{2}, \quad 6 \omega_{2}^{1}+4 \omega_{3}^{2}=12 \omega_{1}^{1}+3 \omega_{4}^{2},
\end{gathered}
$$


Obviously it is difficult to solve the above equations in general. But one can show that these equations hold for graphs which are themselves products of some elementary QD graphs with only one, two or at most three nonzero QD parameters.

Thus, in the following, we will consider two important classes of direct product, namely class $A$ and class $B$ with some relevant examples for each class.

Class A. Class A graphs consist of product of an arbitrary numbers of QD graphs with only two non-vanishing QD parameters

$$
\omega_{1}=a, \quad \omega_{2}=\omega_{3}=\cdots=0 ; \quad \alpha_{1}=0, \quad \alpha_{2}=b, \quad \alpha_{3}=\alpha_{4}=\cdots=0,
$$

where for their $n$-fold direct product we have

$$
\begin{gathered}
\left|\psi_{0}\right\rangle=\left|\phi_{0}\right\rangle\left|\phi_{0}\right\rangle \cdots\left|\phi_{0}\right\rangle=\left|\phi_{0}\right\rangle^{\otimes n} \\
\left|\psi_{1}\right\rangle=\frac{1}{\sqrt{C_{1}^{n}}} \sum_{\text {permutation }}\left|\phi_{0}\right\rangle\left|\phi_{0}\right\rangle \cdots\left|\phi_{0}\right\rangle\left|\phi_{1}\right\rangle, \\
\left|\psi_{2}\right\rangle=\frac{1}{\sqrt{C_{2}^{n}}} \sum_{\text {permutation }}\left|\phi_{0}\right\rangle\left|\phi_{0}\right\rangle \cdots\left|\phi_{0}\right\rangle\left|\phi_{1}\right\rangle\left|\phi_{1}\right\rangle, \\
\vdots \\
\left|\psi_{n}\right\rangle=\left|\phi_{1}\right\rangle\left|\phi_{1}\right\rangle \cdots\left|\phi_{1}\right\rangle=\left|\phi_{1}\right\rangle^{\otimes n} .
\end{gathered}
$$

Application of operators $A^{+}, A^{-}$and $A_{0}$ on quantum states of the product graph given above yields for its QD parameters: $\omega_{k}^{\prime}=k(n-(k-1)) a$, for $k=1,2,3, \ldots, n$, and $\alpha_{k}^{\prime}=(k-1) b$, where the QD property of the product graph imposes the following conditions: $\left|V_{k}\right|-1 \geq \alpha_{k+1}^{\prime}$ and $\frac{\left|V_{k}\right| \alpha_{k+1}^{\prime}}{2}=d$, where $d$ is an integer.

Here we give two non-isomorphic graphs out of many prototypes of class A:

graph1. $\left|V_{k}\right|=\omega_{1}^{\prime} \omega_{2}^{\prime} \ldots, \omega_{k}^{\prime},($ tree $)$

graph $2 .\left|V_{1}\right|=n a, ; \quad\left|V_{2}\right|=2 n^{2}, ; \quad\left|V_{3}\right|=3 \operatorname{an}(n-2), \ldots$. 


\section{$n$-cube as an example of class $\mathrm{A}$}

As an example, we obtain $n$-cube as the direct product of $K_{2}$ graphs by choosing $a=1$, and $b=0$. Hence as we will see in the following that its amplitude $\left|\psi_{t}\right\rangle$ can be written as the product of the $K_{2}$ ones. Since its adjacency matrix can be written as the Cartesian product of the $K_{2}$ graphs therefore

$$
A=\frac{1}{n} \sum_{i=1}^{n} I_{2} \otimes \cdots \otimes \sigma_{x} \otimes \cdots \otimes I_{2}
$$

where the $i$ th term in the sum has $\sigma_{x}$ (Pauli matrix) appearing in the $i$ th place in the tensor product. Therefore, we have

$$
\begin{gathered}
\exp (-i A t)=\prod_{i=1}^{n} I_{2} \otimes \cdots \otimes e^{-i t \sigma_{x} / n} \otimes \cdots \otimes I_{2} \\
=e^{-i t \sigma_{x} / n} \otimes \cdots \otimes e^{-i t \sigma_{x} / n}=\left(e^{-i t \sigma_{x} / n}\right)^{\otimes n}
\end{gathered}
$$

where $B^{\otimes n}$ is the tensor product of $n$ copies of $B$. On the other hand for $K_{2}$ we have

$$
\omega_{1}=1, \quad \omega_{2}=\omega_{3}=\cdots=0, \quad \alpha_{1}=\alpha_{2}=\cdots=0
$$

and

$$
G_{(x)}=\frac{x}{x^{2}-1}, \quad \mu=\frac{1}{2}(\delta(x-1)+\delta(x+1)) .
$$

Hence, for the amplitude of observing the walker at 0 -th stratum of $n$-cube at time $t$, we have

$$
\begin{gathered}
q_{0}(t)=\left\langle\phi_{0}\left|e^{-i t A}\right| \phi_{0}\right\rangle=\left\langle\phi_{0}^{(1)}\left|e^{-i t \sigma_{x} / n}\right| \phi_{0}^{(1)}\right\rangle \cdots\left\langle\phi_{0}^{(n)}\left|e^{-i t \sigma_{x} / n}\right| \phi_{0}^{(n)}\right\rangle \\
=\cos (t / n) \cdots \cos (t / n)=\cos ^{n}(t / n)
\end{gathered}
$$

and the other amplitudes can be calculated by the prescription explained above.

Class B. Class B graphs consist of product of an arbitrary numbers of QD graphs with only four non-vanishing QD parameters

$$
\omega_{1}=\omega_{2}=a, \quad \omega_{3}=\cdots=0 ; \quad \alpha_{1}=0, \quad \alpha_{2}=b, \quad \alpha_{3}=2 b, \quad \alpha_{4}=\alpha_{5}=\cdots=0,
$$


where for their $n$-fold direct product we have

$$
\begin{gathered}
\left|\psi_{0}\right\rangle=\left|\phi_{0}\right\rangle\left|\phi_{0}\right\rangle \cdots\left|\phi_{0}\right\rangle=\left|\phi_{0}\right\rangle^{\otimes n} \\
\left|\psi_{1}\right\rangle=\frac{1}{\sqrt{C_{1}^{n}}} \sum_{\text {permutation }}\left|\phi_{0}\right\rangle\left|\phi_{0}\right\rangle \cdots\left|\phi_{0}\right\rangle\left|\phi_{0}\right\rangle\left|\phi_{1}\right\rangle, \\
\left.\left|\psi_{2}\right\rangle=\frac{1}{\sqrt{C_{1}^{n}+(2 !)^{2} C_{2}^{n}}}\left(2 ! \sum_{\text {permutation }}\left|\phi_{0}\right\rangle\left|\phi_{0}\right\rangle \cdots\left|\phi_{1}\right\rangle\left|\phi_{1}\right\rangle\right)+\sum_{\text {permutation }}\left|\phi_{0}\right\rangle\left|\phi_{0}\right\rangle \cdots\left|\phi_{0}\right\rangle\left|\phi_{2}\right\rangle\right), \\
\vdots \\
\left|\psi_{2 n}\right\rangle=\left|\phi_{2}\right\rangle\left|\phi_{2}\right\rangle \cdots\left|\phi_{2}\right\rangle=\left|\phi_{2}\right\rangle^{\otimes n} .
\end{gathered}
$$

Similarly, application of the operators $A^{+}, A^{-}$and $A_{0}$ on the quantum states of the product graph given above yields for its QD parameters: $\omega_{2 k+1}^{\prime}=(2 k+1)(n-k) a$, for $k=0,1,2,3, \ldots, n$, $\omega_{2 k}^{\prime}=k(2 n-(2 k-1)) a$, for $k=1,2,3, \ldots, n$ and $\alpha_{n+1}^{\prime}=n b$ together with the constraint similar to the class A ones plus an extra constraint $\omega_{1}=\omega_{2}$ coming from its QD property. In the following, out of many prototypes of class B, we give the angular momentum graphs as an example.

\section{Example. Angular momentum graphs}

Angular momentum graphs can be obtained from symmetric tensor product of QD graphs of class A with $a=2$ and $b=1$, called vector graphs ( as the angular momentum 1 can be obtained from symmetric tensor product of vectors). Again the amplitude $\left|\psi_{t}\right\rangle$ can be written as the product of vector graphs, since its adjacency matrix can be written as Cartesian product of vector graphs

$$
A=\frac{1}{n} \sum_{i=1}^{n} I_{7} \otimes \cdots \otimes A^{\prime} \otimes \cdots \otimes I_{7}
$$

where the $i$ th term in the sum has $A^{\prime}$ (adjacency matrix of vector graphs) appearing in the $i$-th place in the tensor product. On the other hand, for vector graph we have

$$
\omega_{1}=\omega_{2}=2, \quad \omega_{3}=\omega_{4}=\cdots=0 ; \quad \alpha_{1}=0, \quad \alpha_{2}=1, \quad \alpha_{3}=2, \quad \alpha_{4}=\alpha_{4}=\cdots=0
$$


and

$G(x)=\frac{x^{2}-3 x}{x^{3}-3 x^{2}-2 x+4}, \quad \mu=\frac{2}{5} \delta(x-1)+\frac{3-\sqrt{5}}{10} \delta(x-(1+\sqrt{5}))+\frac{3+\sqrt{5}}{10} \delta(x-(1-\sqrt{5}))$.

Hence, for $l=n$ angular momentum graph with the following QD parameters

$$
\omega_{1}=2 n, \quad \omega_{1}=2(2 n-1), \cdots, \omega_{k}=k(2 n-k+1), \cdots ; \quad \alpha_{k}=k-1,
$$

the amplitude of observing the walker at 0-th stratum of at time $t$ becomes

$$
\begin{gathered}
q_{0}(t)=\left\langle\phi_{0}\left|e^{-i t A}\right| \phi_{0}\right\rangle=\left\langle\phi_{0}^{(1)}\left|e^{-i t A^{\prime} / n}\right| \phi_{0}^{(1)}\right\rangle \cdots\left\langle\phi_{0}^{(n)}\left|e^{-i t A^{\prime} / n}\right| \phi_{0}^{(n)}\right\rangle \\
=\frac{1}{5} e^{-i t / n}(2+3 \cos (\sqrt{5} t / n)+i \sqrt{5} \sin (\sqrt{5} t / n)) \cdots \frac{1}{5} e^{-i t / n}(2+3 \cos (\sqrt{5} t / n)+i \sqrt{5} \sin (\sqrt{5} t / n)) \\
=\left(\frac{1}{5} e^{-i t / n}(2+3 \cos (\sqrt{5} t / n)+i \sqrt{5} \sin (\sqrt{5} t / n))\right)^{n}
\end{gathered}
$$

and the other amplitudes can be calculated by the prescription explained above.

\section{Some examples of QD graphs}

In this section we provide some examples of finite and infinite QD graphs and using the spectral distribution we calculate the relevant amplitudes of continuous time quantum walks on theses graphs. As far as the authors know, theses examples exhaust all known graphs which have been studied by researcher in quantum walk until now. First we begin with finite QD graphs:

\subsection{Complete graph $K_{n}$}

A complete graph with $\mathrm{n}$ vertices (denoted $\mathrm{Kn}$ ) is a graph with $\mathrm{n}$ vertices in which each vertex is connected to the others (with one edge between each pair of vertices). Therefore, it is a two-stratum QD graph with one non-vanishing QD parameter $\omega_{1}=n-1$ and spectral 
distribution

$$
G_{\mu}(x)=\frac{x-n+2}{x^{2}-(n-2) x-n+1}, \quad \mu=\frac{n-1}{n} \delta(x+1)+\frac{1}{n} \delta(x-(n-1)),
$$

that yields the following amplitudes at time $t$ of the quantum walker

$$
\begin{gathered}
q_{0}(t)=\left\langle\phi_{0}\left|e^{-i A t /(n-1)}\right| \phi_{0}\right\rangle=\int_{R} e^{-i x t /(n-1)} \mu(d x) \\
=\frac{n-1}{n} e^{\frac{i t}{n-1}}+\frac{1}{n} e^{-i t}=\frac{1}{n}\left(e^{-i t}+(n-1) e^{\frac{i t}{n-1}}\right) \\
q_{1}(t)=\left\langle\phi_{1}\left|e^{-i A t /(n-1)}\right| \phi_{0}\right\rangle=\int_{R} \frac{x}{n-1} e^{-i x t /(n-1)} \mu(d x) \\
=\frac{\sqrt{n-1}}{n}\left(e^{-i t}-e^{i t /(n-1)}\right),
\end{gathered}
$$

where the results thus obtained are in agreement with those of Ref.[15].

\subsection{Cycle graph $C_{n}$}

A cycle graph or cycle is a graph that consists of some number of vertices connected in a closed chain. The cycle graph with $\mathrm{n}$ vertices is denoted by $C_{n}$, and quantum walk on them turns out to be different for odd and even $n$, hence we treat them separately.

Odd $n$. For odd $n=2 m+1, C_{2 m+1}$ graph consists of $m+1$ starata, and its QD parameters are

$$
\omega_{1}=2, \quad \omega_{2}=\omega_{3}=\cdots \omega_{m}=1 ; \quad \alpha_{1}=\alpha_{2}=\cdots=0, \quad \alpha_{m+1}=1
$$

It is straightforward to show that it has the following spectral distribution

$$
\mu=\frac{1}{2 m+1} \delta(x-2)+\frac{2}{2 m+1} \sum_{l=1}^{2 m} \delta\left(x-2 \cos \left(\frac{2 l \pi}{2 m+1}\right)\right) .
$$

Therefore, the amplitude probability for walker at time $t$ and 0th stratum is

$$
\begin{aligned}
& q_{0}(t)=\left\langle\phi_{0}\left|e^{-i A t / 2}\right| \phi_{0}\right\rangle=\int_{R} e^{-i x t / 2} \mu(d x) \\
= & \frac{1}{2 m+1} e^{-i t}+\frac{2}{2 m+1} \sum_{l=1}^{m} e^{-i t \cos 2 l \pi /(2 m+1)} .
\end{aligned}
$$


Using Eq.(4-17), one can calculate the other amplitudes, where the results obtained are in agreement with those of Ref.[15].

Even $n$. For even $n=2 m, C_{2 m}$ graph consists of $m+1$ starata, and its QD parameters are

$$
\omega_{1}=2, \quad \omega_{2}=\omega_{3}=\cdots \omega_{m-1}=1 ; \quad \omega_{m}=2 ; \quad \alpha_{1}=\alpha_{2}=\cdots=0 .
$$

One can straightforwardly show that it has the following spectral distribution

$$
\mu=\frac{1}{2 m}(\delta(x-2)+\delta(x+2))+\frac{2}{2 m} \sum_{l=1, l \neq m}^{2 m-1} \delta\left(x-2 \cos \left(\frac{2 l \pi}{2 m}\right)\right) .
$$

Similarly, the amplitude probability for walker at time $t$ and $0-t h$ stratum is

$$
\begin{aligned}
q_{0}(t) & =\left\langle\phi_{0}\left|e^{-i A t / 2}\right| \phi_{0}\right\rangle=\int_{R} e^{-i x t / 2} \mu(d x) \\
& =\frac{1}{m} \cos t+\frac{1}{m} \sum_{l=1}^{m} e^{-i t \cos 2 l \pi /(2 m)} .
\end{aligned}
$$

Again one can calculate the other amplitudes by using Eq.(4-17), where the results are in agreement with those of Ref. [15].

In the limit of large $n$, the roots $x_{l}=2 \cos \left(\frac{2 \pi l}{2 m}\right)$ reduce to $x_{l}=2 \cos (\pi x)$ with $x=$ $\lim _{m, l \rightarrow \infty} \frac{l}{m}$ and both spectral distributions given in (6-48) and (6-51), irrespective of whether $n$ is even or odd, reduce to continuous spectral distribution $\mu(x) d x$ with $\mu(x)$

$$
\begin{aligned}
& \mu(x)=\frac{1}{\pi} \int_{0}^{\pi} d y \delta(x-2 \cos (y)) \\
& =\frac{1}{\pi} \int_{0}^{\pi} d y \frac{\delta(y-\arccos (x / 2))}{2 \sin (y)} \\
& =\frac{1}{\pi} \int_{0}^{\pi} d y \frac{\delta(y-\arccos (x / 2))}{2 \sin (y)} \\
& =\frac{1}{\pi} \frac{1}{\sqrt{4-x^{2}}}, \quad-2 \leq x \leq 2,
\end{aligned}
$$

which is the same as the continuous spectral distribution of infinite line graph Z. 


\subsection{Tchebichef graphs}

By choosing Tchebichef polynomials of first kind (second kind ) with scaling factor $\frac{1}{2^{m}}$ as orthogonal polynomials appearing in recurrence relation (4-11), i.e., $Q_{n}(x)=2^{(m-1) n+1} T_{n}\left(x / 2^{m}\right)$ $\left(2^{(m-1) n} U_{n}\left(x / 2^{m}\right)\right)$, one can obtain a class of finite and infinite QD graphs of Tchebichef type, with QD parameters $\omega_{1}=2^{2(m-1)+1}, \quad \omega_{k}=2^{2(m-1)}, \quad k=2,3, \ldots$, and $\alpha_{k}=0, \quad k=1,2,3, \ldots$ $\left(\omega_{k}=2^{2(m-1)}, \quad k=1,2, \ldots ; \quad \alpha_{k}=0, \quad k=1,2, \ldots\right)$, such that its Stieltjes transform of spectral distribution becomes $G_{\mu}(x)=\frac{1}{n} \frac{T_{n}^{\prime}\left(\frac{x}{2^{m}}\right)}{T_{n}\left(\frac{x}{2^{m}}\right)}\left(\frac{1}{2^{m-1}} \frac{U_{n}\left(\frac{x}{2^{m}}\right)}{U_{n+1}\left(\frac{x}{2^{m}}\right)}\right)$.

Therefore its spectral distribution can be written as $\mu=\frac{1}{n} \sum_{l=0} \delta\left(x-2^{m} \cos \frac{(2 l+1) \pi}{2 n}\right)$ $\left(\frac{1}{n+2} \sum_{l=0} \sin ^{2}\left(\frac{l \pi}{n+2}\right) \delta\left(x-2^{m} \cos \frac{l \pi}{n+2}\right)\right)$, where $2^{m} \cos \frac{(2 l+1) \pi}{2 n}, l=1,2, \ldots\left(2^{m} \cos \left(\frac{l \pi}{n+2}\right), l=1,2, \cdots\right)$ are roots of the Tchebishef polynomial, $T_{n}\left(x / 2^{m}\right)\left(U_{n+1}\left(x / 2^{m}\right)\right)$. Now, using Eq.(4-17) the amplitude of observing walker at different stratum at time $t$ can be calculated as

$$
\begin{gathered}
q_{0}(t)=\frac{1}{n} \sum_{l=0} e^{-i 2^{m} t \cos \left(\frac{(2 l+1) \pi}{2 n}\right)}, \\
q_{k}(t)=\frac{\sqrt{2}}{n} \sum_{l=0} e^{-i 2^{m} t \cos \left(\frac{(2 l+1) \pi}{2 n}\right)} \cos \left(\frac{k(2 l+1) \pi}{2 n}\right), \quad k \geq 1,
\end{gathered}
$$

for the first kind and

$$
\begin{gathered}
q_{0}(t)=\frac{2}{n+2} \sum_{l=0} \sin ^{2}\left(\frac{l \pi}{n+2}\right) e^{-i 2^{m} t \cos \left(\frac{l \pi}{n+2}\right)}, \\
q_{k}(t)=\frac{2}{n+2} \sum_{l=0} \sin ^{2}\left(\frac{l \pi}{n+2}\right) \cos \left(\frac{(k+1) l \pi}{n+2}\right) e^{-i 2^{m} t \cos \left(\frac{l \pi}{n+2}\right)}, \quad k \geq 1,
\end{gathered}
$$

for the second kind.

Finally, in the limit of large $n$, the absolutely continuous part of spectral distribution $\mu(x)$ reads as $\mu(x)=\frac{1}{\pi} \frac{1}{\sqrt{2^{2 m}-x^{2}}}\left(\frac{1}{2 \pi} \sqrt{2^{2 m}-x^{2}}\right)$ such that, $-2^{m} \leq x \leq 2^{m}$ (its derivation is similar to that of cycle graph $\left.C_{n}\right)$ and the amplitude $p_{k}(t)$ given in (6-54) and (6-55) reduce to

$$
\begin{gathered}
q_{0}(t)=J_{0}\left(2^{m-1} t\right) \\
q_{k}(t)=\frac{1}{\sqrt{\omega_{1} \omega_{2} \cdots \omega_{k}}} \int_{-2^{m}}^{2^{m}} 2^{k(m-1)+1} T_{k}\left(x / 2^{m}\right) e^{-i x t / 2} \mu(d x)
\end{gathered}
$$


continuous-time Quantum walk

$$
\begin{gathered}
=\frac{2}{\sqrt{2}}\left[\frac{1}{2 \pi} \int_{-\pi}^{\pi} \cos (k \psi) e^{-i 2^{m-1} t \cos (\psi)} d \psi\right] \\
=\sqrt{2} i^{k} J_{k}\left(2^{m-1} t\right), \quad k \geq 1,
\end{gathered}
$$

for the first kind and

$$
\begin{gathered}
q_{k}(t)=\frac{1}{\sqrt{\omega_{1} \omega_{2} \cdots \omega_{k}}} \int 2^{(m-1) k} U_{k}\left(x / 2^{m}\right) e^{-i x t / 2} \mu(d x) \\
=\frac{1}{2 \pi} \int_{-2^{m}}^{2^{m}} \frac{\sin \left((k+1) \cos ^{-1}\left(x / 2^{m}\right)\right)}{\sqrt{1-\left(x / 2^{M}\right)^{2}}} e^{-i x t / 2} \sqrt{2^{2 m}-x^{2}} d x \\
=\frac{2}{\pi} \int_{0}^{\pi} e^{-i 2^{m} t \cos (\psi)} \sin ((k+1) \psi) \sin (\psi) d \psi \\
=i^{k}\left(J_{k}\left(2^{m} t\right)+J_{k+2}\left(2^{m} t\right)\right), \quad k=0,1,2, \ldots,
\end{gathered}
$$

for the second kind, where $J_{k}(t)$ are the Bessel polynomials. In deriving of (6-56) and (6-57), we have used the integral representation of Bessel function after making the change of variable $x=2^{m} \cos \psi$. Also, one can calculate the amplitudes of infinite Tchebishef graphs by using generating function of Tchebishef and formula Eq.(4-17), where the obtained results are in agreement with those given in (6-56) and (6-57). As we will see in the following, some of the known finite and infinite graphs can be obtained from Tchebishef graphs by appropriate choice of $m$ and the polynomials as:

A. For $m=1$ and Tchebishef polynomial of the second kind we obtain finite path graph $\mathrm{P}_{n}=\{0,1,2, \ldots\}$, where it is a $n$ - vertex graph with $n-1$ edges all on a single open path. Its QD parameters and the amplitude of observing walker at different stratum at time $t$ are

$$
\begin{gathered}
\omega_{1}=\omega_{2}=\omega_{3}=\cdots=1 ; \quad \alpha_{n}=0 \\
q_{0}(t)=\frac{2}{n+2} \sum_{l=1} \sin ^{2}\left(\frac{l \pi}{n+2}\right) e^{-i t \cos \left(\frac{l \pi}{n+2}\right)}, \\
q_{k}(t)=\frac{2}{n+2} \sum_{l=1}^{n+1} \sin (l \pi /(n+2)) \sin \left(\frac{k+1}{n+2} l \pi\right) e^{-i t \cos (l \pi /(n+2))}, \quad k \geq 1,
\end{gathered}
$$


where the results thus obtained are in agreement with those of Ref.[26]. Also, in the limit of large $n, q_{k}(t)$ reduces to

$$
q_{k}(t)=i^{k}\left(J_{k}(t)+J_{k+2}(t)\right), \quad k=0,1, \ldots
$$

B. For $m=\frac{3}{2}$ and Tchebishef polynomial of the second kind we obtain a sequence of graphs $G_{n}$ (for more details see Ref.[7]). The number of vertices in $G_{n}$ is $2^{n+1}+2^{n}-2$. In Fig. 1 we show $G_{4}$. In general, $G_{n}$ consists of two balanced binary trees of depth $n$ with the $2^{n}, n$ th-level vertices of the two trees pairwise identified. For the quantum walk on $G_{n}$, one starts the waker at the root of a tree and wants to calculate the probability of the presence of the walker at the other vertices as function of time. One can show that $G_{n}$ is $\mathrm{QD}$ graph with $(2 n+1)$ strata, where stratum $j$ consists of $2^{j}$ vertices for $j=1,2, \ldots, n+1$ and $2^{(2 n+1-j)}$ for $j=n+1, \ldots, 2 n+1$. Therefore, its QD parameters and the amplitude of observing walker at different stratum at time $t$ are

$$
\begin{gathered}
\omega_{1}=\omega_{2}=\omega_{3}=\cdots=2 ; \quad \alpha_{1}=\alpha_{2}=\cdots=0, \\
q_{0}(t)=\frac{2}{n+2} \sum_{l} \sin ^{2}\left(\frac{l \pi}{n+2}\right) e^{-i 2 \sqrt{2} t \cos \left(\frac{l \pi}{n+2}\right)}, \\
q_{k}(t)=\frac{2}{n+2} \sum_{l=1}^{n+1} \sin (l \pi /(n+2)) \sin \left(\frac{k+1}{n+2} l \pi\right) e^{-i 2 \sqrt{2} t \cos (l \pi /(n+2))}, \quad k \geq 1 .
\end{gathered}
$$

In the limit of large $n, p_{k}(t)$ reduce to

$$
q_{k}(t)=i^{k}\left(J_{k}(2 \sqrt{2} t)+J_{k+2}(2 \sqrt{2} t)\right), \quad k=0,1, \ldots
$$

. C. For $m=1$ and Tchebishef polynomial of the first kind we obtain infinite line graphs $\mathrm{Z}$ where its QD parameters and the amplitude of observing walker at different stratum at time $t$ are

$$
\begin{gathered}
\omega_{1}=2, \quad \omega_{2}=\omega_{3}=\cdots=1 ; \quad \alpha_{k}=0, \\
q_{0}(t)=J_{0}(t),
\end{gathered}
$$


continuous-time Quantum walk

$$
q_{k}(t)=\sqrt{2} i^{k} J_{k}(t), \quad k \geq 1 .
$$

The results thus obtained are in agreement with those of Ref.[27].

\subsection{The finite Hermit graph}

Let $G$ consist of $n$ vertices such that its QD parameters, cardinality of stratums and Stieltjes transform are

$$
\begin{gathered}
\omega_{1}=n, \quad \omega_{2}=n-1, \quad \omega_{3}=n-2, \cdots, \omega_{n}=1, \quad \alpha_{1}=\alpha_{2}=\cdots=0, \\
\left|V_{1}\right|=n, \quad\left|V_{2}\right|=n(n-1), \cdots,\left|V_{n}\right|=n !, \\
G_{\mu}(x)=\frac{H_{n}(x)}{H_{n+1}(x)},
\end{gathered}
$$

where, $H_{n}(x)$ is Hermite polynomials. Then it will have the following spectral distribution

$$
\mu=\frac{1}{n+1} \sum_{l} \delta\left(x-x_{l}\right),
$$

where $x_{l}, l=1,2, \ldots$ are the roots of Hermite polynomial $H_{n}(x)$. Again, using Eq.(4-17) or the recurrence relation (4-18) one can calculate all amplitudes, where in the following we just quote the results for $n=3$. Its QD parameters and spectral distribution are

$$
\begin{aligned}
& \omega_{1}=3, \quad \omega_{2}=2, \quad \omega_{3}=1 ; \quad \alpha_{1}=\alpha_{2}=\cdots=0, \\
& \mu=\frac{1}{4}\left(\delta_{\sqrt{3+\sqrt{6}}}+\delta_{\sqrt{3-\sqrt{6}}}+\delta_{-\sqrt{3+\sqrt{6}}}+\delta_{-\sqrt{3-\sqrt{6}}}\right),
\end{aligned}
$$

and the amplitude as

$$
\begin{gathered}
q_{o}(t)=\frac{1}{2}(\cos (\sqrt{3+\sqrt{6}}) t+\cos (\sqrt{3-\sqrt{6}}) t), \\
q_{1}(t)=\frac{-i}{6}((\sqrt{3+\sqrt{6}}) \sin (\sqrt{3+\sqrt{6}}) t+(\sqrt{3-\sqrt{6}}) \sin (\sqrt{3-\sqrt{6}}) t), \\
q_{2}(t)=\frac{1}{2 \sqrt{6}}(\cos (\sqrt{3+\sqrt{6}}) t-\cos (\sqrt{3-\sqrt{6}}) t), \\
q_{3}(t)=\frac{-i}{6 \sqrt{2}}(\sqrt{3+\sqrt{6}}(\sqrt{3}-\sqrt{2}) \sin (\sqrt{3+\sqrt{6}}) t+\sqrt{3-\sqrt{6}}(\sqrt{3}+\sqrt{2}) \sin (\sqrt{3-\sqrt{6}}) t) .
\end{gathered}
$$




\subsection{The infinite Hermite graph}

By choosing Hermite polynomial $H_{n}(x)$, as orthogonal polynomial appearing in recurrence relation (4-11), one can obtain a class of infinite QD graphs of Hermite type with QD parameters $\omega_{k}=k$, and $\alpha_{k}=0$. Here we give two non-isomorphic graphs out of many prototypes of infinite Hermite graph:

Graph1. $\left|V_{k}\right|=\omega_{1} \omega_{2} \cdots \omega_{k}=k !($ tree $)$ i.e., $\kappa_{-}(y)=1$

Graph2. $\left|V_{1}\right|=1,\left|V_{2}\right|=2\left(\kappa_{-}(y)=1\right),\left|V_{3}\right|=3 !\left(\kappa_{-}(y)=1\right),\left|V_{4}\right|=3 !\left(\kappa_{-}(y)=2\right),\left|V_{5}\right|=$ $30\left(\kappa_{-}(y)=1\right),\left|V_{6}\right|=6 \times 30\left(\kappa_{-}(y)=1\right), \ldots$

Here the amplitude $q_{k}(t)$ can be calculated rather trivially by using generating function of Hermite polynomials as follows:

$$
\begin{gathered}
\sum_{k=0} \sqrt{\frac{1}{2 k ! \pi}} q_{k}(t) y^{k}=\int_{R} e^{-i x t} \sum_{k} \frac{y^{k}}{k !} H_{k}(x) \mu(d x)=\int_{-\infty}^{\infty} e^{-i x t} e^{x y-y^{2} / 2} e^{-x^{2} / 2} d x \\
=e^{-y^{2} / 2} \int_{-\infty}^{\infty} e^{-1 / 2\left(x^{2}+2 x(i t-y)\right)} d x=e^{-\frac{1}{2} t(t+2 i y)}
\end{gathered}
$$

Now, by comparing the terms of the expansion appearing on the left hand side of (6-67) with those of expansion on the right hand side we obtain

$$
q_{k}(t)=\frac{(-i)^{k} t^{k}}{\sqrt{k !}} e^{-t^{2} / 2}
$$

where the result thus obtained is exactly the same as that of WKB approximation, i.e, the method of stationary phase gives the exact result in infinite Hermite case. Then, the probability distribution $\left|q_{k}(t)\right|^{2}=\frac{\left(t^{2}\right)^{k}}{k !} e^{-t^{2}}$ is commonly called the Poisson distribution.

\subsection{The infinite Laguerre graph}

We take the $Q_{n}(x)$ as a Laguerre polynomial, such that they satisfy the orthogonal polynomials appearing in the recurrence relation (4-11), i.e, $Q_{n}(x)=n !(-1)^{n} a^{n} L_{n}^{(\gamma)}\left(\frac{x-b}{a}\right)$. Then, one can obtain a class of infinite QD graphs of Laguerre type, with QD parameters $\omega_{k}=a^{2} k(k+\gamma)$, and choose $b=-a(1+\gamma), \alpha_{1}=0$ and $\alpha_{k+1}=2 k a$ for $k \geq 1$. Here we give two non-isomorphic 
graphs out of many prototypes of infinite Hermite graph:

Graph1. $\left|V_{k}\right|=\omega_{1} \omega_{2} \cdots \omega_{k}($ tree $)$ i.e., $\kappa_{-}(y)=1$

Graph2. $\left|V_{k}\right|=k ! a^{2}(1+\gamma)(2+\gamma) \cdots(k+\gamma), \quad\left(\kappa_{-}(y)=a\right)$.

By using Eq.(4-17), we obtain generating functions for amplitude probability $q_{k}(t)$

$$
\begin{gathered}
\sum_{k=0} \sqrt{\frac{(1+\gamma)(2+\gamma) \cdots(k+\gamma)}{k !}} q_{k}(t) y^{k}=\int_{R} e^{-i x t} \sum_{k}(-1)^{k} a^{k} k ! L_{k}^{\gamma}\left(\frac{x-b}{a}\right) \frac{y^{k}}{k !} \mu(d x) \\
=\frac{1}{a} \int_{0}^{\infty} e^{-i x t} \frac{-\frac{y(x-b)}{a(1-y)}}{(1-y)^{\gamma+1}}\left(\frac{x-b}{a}\right)^{\gamma} e^{-\left(\frac{x-b}{a}\right)} d x=\frac{\gamma ! e^{-i b t}}{(1+i a(1-y) t)^{\gamma+1}} .
\end{gathered}
$$

It is straightforward to show that $p_{k}(t)$ has the following form

$$
q_{k}(t)=\sqrt{\frac{(\gamma+k) !}{k ! \gamma !}} \frac{e^{-i b t}(i a t)^{k}}{(1+i a t)^{k+\gamma+1}} .
$$

Formula (6-70) implies that for distant time the amplitude becomes proportional to the inverse

of time $t$, i.e, we have $q_{k}(t) \approx \sqrt{\frac{(\gamma+k) !}{k ! \gamma !}} \frac{e^{-i b t}}{t^{\gamma+1}}$, which is the same as the one obtained by WKB approximation.

\section{Asymptotic behavior of continuous-time quantum walk in the large time limit}

\subsection{Average moments of number of visiting strata}

Studying the large time behavior of moments and variance as function of $t$ for classical random walk and quantum walk is important, since common properties for describing the behavior of a walker are the exponents $\nu$, characterizing the scaling with time t of the mean square end-toend distance $\left\langle R^{2}\right\rangle=\sqrt{\left\langle k^{2}\right\rangle-\langle k\rangle^{2}} \approx t^{\nu}$ (the exponent $\nu$ determine universality class). The walk on infinite line, $\left|q_{k}(t)\right|^{2}$ has the form of a binomial distribution, with a width which spreads like $t^{1 / 2}($ i.e, $\nu=1 / 2)$, therefore the variance grows linearly with time. But the variance in the quantum walk on infinite line, by contrast, grows quadratically with time, and the distribution 
$\left|q_{k}(t)\right|^{2}$ has a complicated, oscillatory form[28]. Now, by using the probability amplitudes of Eq.(6-68) and Eq.(6-70) we evaluate the average of different moments of stratum number, i.e, $\left\langle k^{q}\right\rangle$ ( $q$-th moment, $\left.q=1,2,3, \ldots\right)$, for quantum walk on infinite Hermite, Laguerre and line graphs as follows:

A. Using the generating function Hermite polynomials, the average $q$-moment of infinite Hermite graphs can be written as

$$
\left\langle k^{q}\right\rangle=\sum_{k=0}^{\infty} k^{q} \frac{\left(t^{2}\right)^{k}}{k !} e^{-t^{2}}=e^{-t^{2}}\left(t^{2} \frac{d}{d\left(t^{2}\right)}\right)^{q} \sum_{k=0}^{\infty} \frac{\left(t^{2}\right)^{k}}{k !}=e^{-t^{2}}\left(t^{2} \frac{d}{d\left(t^{2}\right)}\right)^{q} e^{t^{2}}
$$

Above formula implies that $\langle k\rangle=t^{2}$ and $\left\langle k^{2}\right\rangle=t^{4}+t^{2}$, therefore for it standard deviation $\sigma(t)$ we get $\sigma(t)=\sqrt{\left\langle k^{2}\right\rangle-\langle k\rangle^{2}}=t$. Also, it is straightforward to see that for large time, the $q$-moment has the following power dependence upon time

$$
\left\langle k^{q}\right\rangle \approx\left(t^{2}\right)^{q}
$$

B. Similarly for infinite Laguerre graphs we have

$$
\left\langle k^{q}\right\rangle=\sum_{k=0}^{\infty} k^{q} \frac{(\gamma+k) !}{k ! \gamma !} \frac{(a t)^{2 k}}{\left(1+(a t)^{2}\right)^{k+\gamma+1}}=\left.\frac{1}{\left(1+a^{2} t^{2}\right)^{\gamma+1}}\left(y \frac{d}{d y}\right)^{q}\left(\frac{1}{(1-y)^{\gamma+1}}\right)\right|_{y=\frac{a^{2} t^{2}}{1+a^{2} t^{2}}}
$$

where it implies that

$$
\begin{gathered}
\langle k\rangle=(\gamma+1) a^{2} t^{2}, \quad\left\langle k^{2}\right\rangle=(\gamma+1) a^{2} t^{2}+(\gamma+1)(\gamma+2)\left(a^{2} t^{2}\right)^{2} \\
\lim _{t \rightarrow \infty}(\sigma(t))=t^{2}, \quad \lim _{t \rightarrow \infty}\left\langle k^{q}\right\rangle \approx\left(a^{2} t^{2}\right)^{q} .
\end{gathered}
$$

C. In infinite line graph, where the walker starts at origin, we calculate the average moments of visiting number of sites (therefore $k$ denotes the sites of graph where $k=0$ correspond to its origin). again using the well known the generating Bessel function we can show that

$$
\begin{gathered}
\langle k\rangle=\sum_{k=-\infty}^{\infty} 2 k\left|J_{k}(t)\right|^{2}=0, \\
\left\langle k^{2}\right\rangle=\sum_{k=-\infty}^{\infty} 2 k^{2}\left|J_{k}(t)\right|^{2}=t^{2},
\end{gathered}
$$




$$
\begin{gathered}
\lim _{t \rightarrow \infty}\left\langle k^{q}\right\rangle==\left\{\begin{array}{cc}
t^{q} & \text { for } q=\text { even } \\
0 & \text { otherwise. }
\end{array}\right. \\
\sigma(t) \approx t .
\end{gathered}
$$

In the above examples the the average number of visiting strata (sites for infinite line), the quantity, $\sigma(t)$ is the same as end-to-end distance. The end-to-end distance for quantum walker on infinite Laguerre graphs varies quadratically with time, i.e, $\left\langle R^{2}\right\rangle \approx t^{2}$, while the end-toend distance for walker on infinite Hermite graphs is $\left\langle R^{2}\right\rangle \approx t$. Thus, the quantum walk on infinite line and Hermite graphs have the same critical exponent, which is different from infinite Laguerre one. Therefore, quantum walk on infinite line and Hermite graphs belong to the same universality class, which is different from the universality class of quantum walk on infinite Laguerre graph.

\subsection{Asymptotic behavior of the probability distribution in the large time limit}

In general we can not have an analytic expression for the amplitudes of continuous time quantum walk on most of graphs, i.e, the integral appearing in the Eq.(4-17) is difficult to evaluate, but one can approximate it for large time by using the method of stationary phase explained above in section 4 . In order to see the importance of spectral distribution method, we obtain the asymptotic behavior of some finite and infinite graphs in remaining part of this section.

\subsubsection{The infinite graphs:}

\subsubsection{Star lattice}

Star lattice is an $N$-fold star power $G^{\star N}$, where $G=(V, E)$ is the half line of integer, i.e., $V=$ $0,1,2, \ldots$ and $i \sim j$ if and only if $|i-j|=1$, so its QD parameters are $\omega_{1}=N, \omega_{k}=1, \alpha_{k}=0$ 
for $k=2,3, \ldots$, and as it is shown in Ref.[19] it as the following spectral distribution

$$
\mu(x)=\frac{1}{2 \pi} \frac{N \sqrt{4-x^{2}}}{N^{2}-(N-1) x^{2}}, \quad-2 \leq x \leq 2 .
$$

Therefore, its ground stratum amplitude $q_{0}(t)$ can be written as

$$
q_{0}(t)=\int_{-2}^{2} e^{-i t x} \mu(d x)=\int_{-2}^{2} e^{-i t x}(x) \frac{1}{2 \pi} \frac{N \sqrt{4-x^{2}}}{N^{2}-(N-1) x^{2}} d x
$$

Now, its asymptotic form can be obtained by the method of stationary phase after making the change of variable $x=2 \cos (\theta)$, where we get

$$
q_{0}(t) \approx \frac{4 N \Gamma(3 / 2)}{\pi(N-2)^{2}} \frac{1}{\sqrt{t}} \cos \left(2 t-\frac{3 \pi}{4}\right)
$$

for $N \neq 2$, while, for $N=2$ the star lattices reduces to infinite line graphs with spectral distribution $\mu(x)=\frac{1}{\pi} \frac{1}{\sqrt{4-x^{2}}},-2 \leq x \leq 2$ and asymptotic ground amplitude $q_{0}(t)=J_{0}(2 t) \approx$ $\frac{1}{\sqrt{\pi t}} \cos \left(2 t-\frac{\pi}{4}\right)$

\subsubsection{Two-dimensional comb lattice}

As another example we consider two-dimensional comb lattice of Ref. [29], with the following spectral distribution

$$
\mu(x) d x=\frac{1}{\pi} \frac{d x}{\sqrt{8-x^{2}}}, \quad-2 \sqrt{2} \leq x \leq 2 \sqrt{2}
$$

and ground stratum amplitude

$$
\begin{gathered}
q_{0}(t)=\int_{-2 \sqrt{2}}^{2 \sqrt{2}} e^{-i t x / 4} \rho(x) d x=\int_{-2 \sqrt{2}}^{2 \sqrt{2}} \frac{1}{\pi} \frac{e^{-i t x / 4} d x}{\sqrt{8-x^{2}}} \\
=\frac{1}{\pi} \int_{0}^{\pi} e^{-i t \cos \theta / \sqrt{2}} d \theta
\end{gathered}
$$

Again its asymptotic form can be obtained by the method of stationary phase after making the change of variable $x=2 \sqrt{2} \cos (\theta)$, where we get

$$
q_{0}(t) \sim \sqrt{\frac{2 \sqrt{2}}{\pi t}} \cos (t / \sqrt{2}-\pi / 4) .
$$


7.2.2 The finite graphs: In order to obtain the asymptotic form of finite graphs at large times, we need to use Gauss quandrature formula(4-21) to approximate finite graphs with the infinite ones and then using the method of stationary phase we can obtain their asymptotic form. In the example under discussion asymptotic behavior of quantum walk on line and generalized to asymptotic behavior quantum walk on finite graphs. For line $q_{0}(t)$ is $J_{0}(t)$, then for large time $t$ we have $q_{0}(t) \approx \sqrt{\frac{2}{t \pi}} \cos \left(t-\frac{\pi}{4}\right)$. If we use (4-21) and calculate $q_{0}(t)$ on finite graph line we have $q_{0}(t)=\frac{1}{n} \sum_{k=0}^{n-1} e^{-i t \cos \left(\frac{2 k+1}{2 n} \pi\right)}$. Now, we calculate numerically the difference of amplitudes of infinite line with finite graphs $\pi(n, t)$, for large time $t$

$$
\begin{gathered}
\pi(n, t)=\left|J_{0}(t)-\frac{1}{n} \sum_{k=0}^{n-1} e^{-i t \cos \left(\frac{2 k+1}{2 n} \pi\right)}\right| \\
=\left|\sqrt{\frac{2}{t \pi}} \cos \left(t-\frac{\pi}{4}\right)-\frac{1}{n} \sum_{k=0}^{n-1} e^{-i t \cos \left(\frac{2 k+1}{2 n} \pi\right)}\right| .
\end{gathered}
$$

The results are depicted in Fig. 2 and Fig.3, where $\pi(n, t)$ is in the limit $n$ from 500 to 600 and $t$ in the limited 1000, is limited to zero. Then, to study the behavior of asymptotic quantum walk on finite graph line, we study the behavior of asymptotic quantum walk on infinite graph line. Therefore to study the behavior of the asymptotic quantum walk on finite graphs, we can use arithmetic, approximate it with infinite graph, and by using the method of stationary phase, study the behavior of asymptotic quantum walk.

\section{Conclusion}

Using the spectral distribution associated with the adjacency matrix, a new formalism for investigation of continuous-time quantum walk on some graphs is developed, where the orthogonal polynomial together with their recursion relations play an important role. As as by product, by using the Gaus quadrature formula one can approximate infinite graph with finite one and vice versa, which leads to the derivation of large time asymptotic form of continuoustime quantum walk amplitudes of both finite and infinite graph, simply by using the method 
of stationary phase. Even though the powerful method of spectral distribution seems to work for some restricted kinds of graphs introduced in Ref. [19], but it is possible to generalize it to work for most of graphs, particularly for the graphs with strata dependent $\kappa_{-}(y)$ and regular distant ones, which is under investigation.

\section{Appendix A}

In this appendix we prove the following lemma in connection with the equality of continuoustime quantum walk amplitudes on the vertices belonging to the same stratum.

Lemma 1. Let $q_{i k}(t)$ denote the amplitude of observing the continuous-time quantum walker at vertex $i \in V_{k}$ at time $t$. Then for a class of QD graphs, the amplitude $q_{i k}$ is the same for all vertices of stratification $k$, for all $t$.

Proof.

Let as take the Fourier transform of unit vectors $|k, i\rangle$ of the stratum $V_{k}$ :

$$
\left|\phi_{k, l}\right\rangle=\frac{1}{\sqrt{\left|V_{k}\right|}} \sum_{i=0}^{\left|V_{k}\right|-1} \omega_{\left|V_{k}\right|}^{i l}|k, i\rangle
$$

where $\omega_{\left|V_{k}\right|}=e^{\frac{2 \pi i}{\left|V_{k}\right|}}$. Now, acting the adjacency matrix $A$ on it according to the formula (3-4), we obtain

$$
A\left|\phi_{k, 0}\right\rangle=\sqrt{\omega_{k+1}}\left|\phi_{k+1,0}\right\rangle+\alpha_{k+1}\left|\phi_{k, 0}\right\rangle+\sqrt{\omega_{k}}\left|\phi_{k-1,0}\right\rangle
$$

Therefore, $\left\langle\phi_{m, l}|A| \phi_{k, 0}\right\rangle=0$, for $l \neq 0$, hence we have

$$
\begin{gathered}
\left\langle\phi_{k, l}\left|e^{-i A t}\right| \phi_{0, l}\right\rangle=\frac{1}{\sqrt{\left|V_{k}\right|}} \sum_{i \in V_{k}} \omega_{\left|V_{k}\right|}^{i l} p_{i k}(t)=0, \quad \text { for }, l \neq 0, \\
q_{k}(t)=\frac{\left.p_{0 k}(t)+p_{1 k}(t)+p_{2 k}(t)+\cdots+p_{\left(\left|V_{k}\right|\right) k}-1\right)(t)}{\left|V_{k}\right|} \quad \text { for }, l=0,
\end{gathered}
$$


or

$$
F\left(\begin{array}{c}
q_{0 k}(t) \\
q_{1 k}(t) \\
q_{2 k}(t) \\
\vdots \\
q_{\left(\left|V_{k}\right|-1\right) k}(t)
\end{array}\right)=\left(\begin{array}{c}
q_{k}(t) \\
0 \\
0 \\
\vdots \\
0
\end{array}\right)
$$

where $\left|V_{k}\right| \times\left|V_{k}\right|$ the discrete Fourier transformation matrix(DFT) $F$ is defined as

$$
F=\frac{1}{\sqrt{\left|V_{k}\right|}}\left(\begin{array}{lllll}
1 & 1 & 1 & \ldots & 1 \\
1 & \omega & \omega^{2} & \ldots & \omega^{\left|V_{n}\right|-1} \\
1 & \omega^{2} & \omega^{4} & \ldots & \omega^{2\left(\left|V_{n}\right|-1\right)} \\
\vdots & \vdots & \vdots & & \vdots \\
1 & \omega^{\left|V_{n}\right|-1} & \omega^{2\left(\left|V_{n}\right|-1\right)} & \ldots & \omega^{\left(\left|V_{n}\right|-1\right)^{2}}
\end{array}\right)
$$

Inverting $F$ in Eq.(A-iv) we obtain $q_{0 k}(t)=q_{1 k}(t)=\cdots=q_{\left(\left|V_{k}\right|-1\right) k}(t)=\frac{q_{k}(t)}{\left|V_{k}\right|}$.

\section{Appendix B}

\section{list of orthogonal polynomials connected with some finite and infinite graphs}

In this appendix we give a list of orthogonal polynomials (in addition to those mentioned above in section 6), where after appropriate one-dimensional, affine transformation(shift and recalling), they can be reduced to monic orthogonal polynomials with recursion relation(4-11), such that the corresponding parameters $\omega_{n}$ and $\alpha_{n}$ can be connected with some finite or infinite graphs of QD type.

\section{Charlier polynomials}

$Q_{n}(x)=a^{n} C_{n}^{(d)}\left(\frac{x-b}{a}\right), \quad \mu(x)=\sum_{j=0} \frac{e^{-d} d^{x}}{x !} \delta(x-j), \quad$ interval $=(0,+\infty), \quad b=$ $-a d, \quad \omega_{n}=a^{2} n d, \quad \alpha_{n+1}=n a$.

Graph1. $\left|V_{n}\right|=\omega_{1} \omega_{2} \cdots \omega_{n}($ tree $)$,

Graph2. $\left|V_{n}\right|=n ! a^{2} d^{n}$ and $\kappa_{-}(y)=a$. 


\section{Meixner polynomials of the second kind}

$Q_{n}(x)=a^{n} M_{n}\left(\frac{x-b}{a}\right), \quad \mu(x)=\mu(x ; \delta, \eta)=(\Gamma(\eta / 2))^{-2}\left|\Gamma\left(\frac{\eta+i x}{2}\right)\right|^{2} e^{-x \tan ^{-1}(\delta)}, \quad$ interval $=$ $(-\infty,+\infty), \quad b=-a \eta \delta, \quad \omega_{n}=a^{2} n(n+\eta+1)\left(\delta^{2}+1\right), \quad \alpha_{n+1}=2 a n \delta$.

Graph1. $\left|V_{n}\right|=\omega_{1} \omega_{2} \cdots \omega_{n}($ tree $)$,

Graph2. $\left|V_{n}\right|=n ! a^{2}(2+\eta)(3+\eta) \cdots(n+1+\eta)$ and $\kappa_{-}(y)=a$.

\section{Orthogonal polynomials related to Jacobi Elliptic functions}

\section{1}

$Q_{n}(x)=a^{n} A_{n}\left(\frac{x-b}{a}\right), \quad \mu(x)=\sum_{j=0}^{+\infty} \frac{k K^{2}}{\pi^{2}(2 n) !(2 n+1) ! k^{2 n}} \frac{(2 j+1) q^{(2 j+1) / 2}}{1-q^{(2 j+1)}} \delta(x-j), \quad$ interval $=$ $(0,+\infty), \quad b=-a\left(1+k^{2}\right), \quad \omega_{n}=4 a^{2} n^{2}\left(4 n^{2}-1\right) k^{2}, \quad \alpha_{n+1}=4 a n(n+1)\left(1+k^{2}\right)$.

Graph1. $\left|V_{n}\right|=\omega_{1} \omega_{2} \cdots \omega_{n}($ tree $)$,

Graph2. $\left|V_{n}\right|=4^{n}(n !)^{2} a^{2}\left(1 \times 3 \times 15 \times \ldots \times\left(4 n^{2}-1\right)\right) k^{2 n}$ and $\kappa_{-}(y)=a$.

$Q_{n}(x)=a^{n} B_{n}\left(\frac{x-b}{a}\right), \quad \mu(x)=\sum_{j=1}^{+\infty} \frac{k^{2} K^{4}}{2 \pi^{4}(2 n+1) !(2 n+2) ! k^{2 n}} \frac{j^{3} q^{j}}{1-q^{2 j}} \delta(x-j), \quad$ interval $=(1,+\infty), \quad b=$ $-4 a\left(1+k^{2}\right), \quad \omega_{n}=a^{2} 4 n(n+1)(2 n+1)^{2} k^{2}, \quad \alpha_{n+1}=4 a n(n+2)\left(1+k^{2}\right)$.

Graph1. $\left|V_{n}\right|=\omega_{1} \omega_{2} \cdots \omega_{n}($ tree $)$,

Graph2. $\left|V_{n}\right|=4^{n} n !(n+1) ! a^{2}\left(3^{2} \times 5^{2} \times \ldots \times(2 n+1)^{2}\right) k^{2 n}$ and $\kappa_{-}(y)=a$.

$Q_{n}(x)=C_{n}(x), \quad \mu(x)=\sum_{j=-\infty}^{+\infty} \frac{\pi}{k K(n) !^{2} k^{2[n / 2]}} \frac{\left(q^{(2 j+1) / 2}\right.}{1+q^{(2 j+1)}} \delta(x-j), \quad$ interval $=(-\infty,+\infty), \quad b=$ $-4 a\left(1+k^{2}\right), \quad \omega_{2 n+1}=(2 n+1)^{2}, \quad \omega_{2 n}=(2 n)^{2} k^{2}, \quad \alpha_{n}=0$.

Graph1. $\left|V_{n}\right|=\omega_{1} \omega_{2} \cdots \omega_{n}($ tree $)$.

3.4

$Q_{n}(x)=D_{n}(x), \quad \mu(x)=\sum_{j=-\infty}^{+\infty} \frac{\pi}{K(n) !^{2} k^{2[(n+1) / 2]}} \frac{q^{j}}{1+q^{2 j}} \delta(x-j), \quad$ interval $=(-\infty,+\infty), \omega_{2 n+1}=$ $(2 n+1)^{2} k^{2}, \quad \omega_{2 n}=(2 n)^{2}, \quad \alpha_{n}=0$.

Graph1. $\left|V_{n}\right|=\omega_{1} \omega_{2} \cdots \omega_{n}($ tree $)$.

Such that, $j=0, \pm 1, \pm 2, \cdots, \quad K=\int_{0}^{\infty}\left(1-k^{2} \sin ^{2}(\phi)\right)^{-1 / 2} d \phi$ and $q$ is a certain constant related to $k$ which appears in the theory of theta function. 


\subsection{Carlitz polynomials}

$Q_{n}(x)=a^{n} F_{n}\left(\frac{x-b}{a}\right), \quad b=-a, \quad \omega_{n}=4 a^{2} n^{2}(2 n-1)^{2} k^{2}, \quad \alpha_{n+1}=4 a n\left(n\left(1+k^{2}\right)+1\right)$.

Graph1. $\left|V_{n}\right|=\omega_{1} \omega_{2} \cdots \omega_{n}($ tree $)$,

Graph2. $\left|V_{n}\right|=4^{n} a^{2} n !^{2}\left(1 \times 3^{2} \times 5^{2} \times \ldots \times(2 n-1)^{2}\right) k^{2 n}$ and $\kappa_{-}(y)=a$.

3.5 .2

$Q_{n}(x)=a^{n} G_{n}\left(\frac{x-b}{a}\right), \quad b=-a k^{2}, \quad \omega_{n}=4 a^{2} n^{2}(2 n-1)^{2} k^{2}, \quad \alpha_{n+1}=4 a n\left(n\left(1+k^{2}\right)+k^{2}\right)$.

Graph1.| $V_{n} \mid=\omega_{1} \omega_{2} \cdots \omega_{n}($ tree $)$,

Graph2. $\left|V_{n}\right|=4^{n} a^{2} n !^{2}\left(1 \times 3^{2} \times 5^{2} \times \ldots \times(2 n-1)^{2}\right) k^{2 n}$ and $\kappa_{-}(y)=a$.

$Q_{n}(x)=a^{n} G_{n}^{\star}\left(\frac{x-b}{a}\right), \quad b=-a\left(4+k^{2}\right), \quad \omega_{n}=4 a^{2} n^{2}(2 n+1)^{2} k^{2}, \quad \alpha_{n+1}=4 a n(n+1)\left(1+k^{2}\right)$.

Graph1. $\left|V_{n}\right|=\omega_{1} \omega_{2} \cdots \omega_{n}($ tree $)$,

Graph2. $\left|V_{n}\right|=4^{n} a^{2} n !^{2}\left(1 \times 3^{2} \times 5^{2} \times \ldots \times(2 n-1)^{2}\right) k^{2 n}$ and $\kappa_{-}(y)=a$.

The explicit weight function for the subsection (3.5) can be obtained routinely from the weight function of the subsections (3.3) and (3.4).

\section{References}

[1] P. Diaconis(1988), Group Representation in Probability and Statistics, Hayward, California: Institute of Mathematical Statistics.

[2] L. Lovasz, Random Walks on Graph: A Survey, in Combinatorics: Paul Erdos is Eighty, volume 2, edited by D. Miklos, V.T. Sos, and T. Szonyi (Budapest: Janos Bolyai Mathematical Society, 1996), 353-398.

[3] R. Motwani, and P. Raghvan(1995), Randomized Algorithms, Combridge University Press. 
[4] I. Chuang and M. Nielsen (2000), Quantum Information and Quantum Computation, Cambridge University Press.

[5] P. Diaconis (1988), Group Representations in Probability and Statistics, Institute of Mathematical Statistics.

[6] E. Farhi and S. Gutmann (1998), Quantum Computation and Decision Trees, Phys. Rev. A 58.

[7] E. Farhi, M. Childs, and S. Gutmann(2002), An example of the Difference between Quantum and Classical Random Walks, Quantum Information Processing, vol.1, p.35.

[8] A. Ambainis, E. Bach, A. Nayak, A. Viswanath, and J. Watrous (2001), One-Dimensional Quantum Walks, in Proceedings of the 33rd ACM Annual Symposium on Theory Computing (ACM Press), p. 60.

[9] D. Aharonov, A. Ambainis, J. Kempe, and U. Vazirani (2001), Quantum Walks on Graphs, in Proceedings of the 33rd ACM Annual Symposium on Theory Computing (ACM Press), p. 50 .

[10] C. Moore and A. Russell (2002), Quantum Walks on the Hypercube, in Proceedings of the 6th Int. Workshop on Randomization and Approximation in Computer Science (RANDOM'02).

[11] J. Kempe (2003), Discrete Quantum Random Walks Hit Exponentially Faster, Proceedings of 7th International Workshop on Randomization and Approximation Techniques in Computer Science (RANDOM'03), p. 354-69.

[12] R. Feynman, R. Leighton, and M. Sands(1965), The Feynman Lectures on Physics, Volume 3, Addison-Wesley. 
[13] Y. Aharonov, L. Davidovich, and N.Zagury(1993), Quantum Randoms Walk, Phy. Rev. lett 48, p.1687-1690.

[14] A. Childs, E. Deotto, R. Cleve, E. Farhi, S. Gutmann, D. Spielman (2003), Exponential Algorithmic Speedup by Quantum Walk, in Proc. 35th Ann. Symp. Theory of Computing (ACM Press), p. 59.

[15] A. Ahmadi, R. Belk, C. Tamon and C. Wendler(2003), On Mixing in Continuous-Time Quantum Walks on some Circulant Graphs, Quantum Information and Computation, Vol. 3, No. 6, p. 611-618.

[16] W. Adamczak, K. Andrew, P. Hernberg, and C. Tamon(2003), A Note on Graphs Resistant to Quantum Uniform Mixing, in quant-ph/0308073.

[17] H. Gerhardt, and J. Watrous(2003), Continuous-Time Quantum Walks on the Symmetric Group, Proceedings of 7th International Workshop on Randomization and Approximation Techniques in Computer Science (RANDOM'03), p. 290-301.

[18] A. Hora, and N. Obata(2002), An Interacting Fock Space with Periodic Jacobi Parameter Obtained from Regular Graphs in Large Scale Limit, to appear in: Quantum Information V, Hida, T., and Saito , K., Ed., World Scientific, Singapore.

[19] N. Obata(2004), Quantum Probabilistic Approach to Spectral Analysis of Star Graphs, Interdisciplinary Information Sciences, Vol. 10, No. 1, p. 41-52.

[20] T. S. Chihara, An Introduction to Orthogonal Polynomials, Gordon and Breach, Science Publishers Inc(1978).

[21] J. A. Shohat, and J. D. Tamarkin, The Problem of Moments, American Mathematical Society, Providence, RI (1943). 
[22] A. Hora, and N. Obata, Quantum Decomposition and Quantum Central Limit Theorem, in: Fundamental Problems in Quantum Physics, Accardi, L., and Tasaki, S., Ed., p. 284305, World Scientific, Singapore(2003).

[23] M. A. Jafarizadeh, and H. Fakhri(1997), Supersymmetry and Shape Invariance in Differential Equations of Mathematical Physics, Physics letters A, 230, p.164.

[24] Carl M. Bender, and Steven A. Orszag, Advanced Mathematical Methods for Scientists and Engineers, International Series in Pure and Applied Mathematics. McGraw-Hill, Inc., New $\operatorname{York}(1978)$.

[25] N. Bleistein, and Richard A. Handelsman, Asymptotic Expansions of Integrals, Holt, Rinehart and Winston, New York (1975).

[26] D. Ben-Avraham, E. Bollt, and C. Tamon(2004), One-Dimensinal Continuous-Time Quantum Walk, Quantum Information Processing, Vol. 3, p. 295-308.

[27] N. Konno(2005), Continuous-Time Quantum Walk on the Line, in quan-ph/0408140.

[28] T. A. Brun, H. A. Carteret, and A. Ambainis(2003), Quantum Random Walks with Decoherent Coins, Phys. Rev. A 67, 032304.

[29] L. Accardi, ANIS B. Ghorbal, and N. Obata(2004), Monotone Independence, Comb Graphs and Bose-Einstein Condensation, Infnite Dimensional Analysis, Quantum Probability and Related Topics Vol. 7, No. 3, 419-435. 


\section{Figure Captions}

Figure-1: Figure $G_{4}$.

Figure-2: Shows $\pi(n, t)$ for the infinite line graphs with the $n$-vertices finite graph (obtain from the infinite on via Gauss quadrature rule) as a function $n$ at $t=1000$, where the difference is almost negligible for $n \geq 518$.

Figure-3: Figure3 show $\pi(n, t)$ for the infinite line graph with the $n$-vertices finite graph as a function of time and number of vertices $n$, where the difference is almost disappear for $t \geq 1000$ and $n \geq 518$. 